\title{
Trastuzumab-Based Combination Chemotherapy in Patients with Human Epidermal Growth Factor Receptor-2-Positive Metastatic Carcinoma ex Pleomorphic Adenoma
}

Yohei Arihara ${ }^{a, b} \quad$ Kazuyuki Murase ${ }^{a, c} \quad$ Kohichi Takadaa, c Naotaka Hayasaka ${ }^{a}$ Shogo Miura ${ }^{a}$ Koji Miyanishia Masayoshi Kobune ${ }^{c}$ Makoto Kurose $^{d}$ Yukinori Akiyama ${ }^{e}$ Shintaro Sugita ${ }^{f}$ Junji Kato ${ }^{a}$

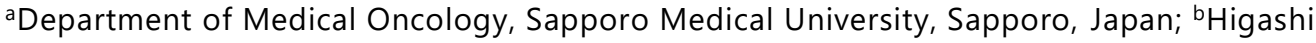
Sapporo Hospital, Sapporo, Japan; 'Department of Hematology, Sapporo Medical University, Sapporo, Japan; dDepartment of Otorhinolaryngology, Sapporo Medical University, Sapporo, Japan; eDepartment of Neurosurgery, Sapporo Medical University, Sapporo, Japan; fDepartment of Surgical Pathology, Sapporo Medical University, Sapporo, Japan

\section{Keywords}

HER2 - Carcinoma ex Pleomorphic Adenoma - CXPA - Trastuzumab - Nab-paclitaxel

\section{Abstract}

Background: Carcinoma ex pleomorphic adenoma (CXPA) is a rare histologic subtype of lacrimal gland and submandibular gland cancer. Currently, there is no standard treatment for metastatic CXPA, although some case reports have explored the role of targeted agents in 


\section{Case Reports in Oncology}

Case Rep Oncol 2018;11:835-841

DOI: $10.1159 / 000495344$

(C) 2018 The Author(s). Published by S. Karger AG, Base www.karger.com/cro

Arihara et al.: Trastuzumab-Based Combination Chemotherapy in Patients with Human Epidermal Growth Factor Receptor-2-Positive Metastatic Carcinoma ex Pleomorphic Adenoma

chemotherapy. A few histopathologic analyses have shown that some of these tumors overexpress human epidermal growth factor receptor-2 (HER2), suggesting a potential role for HER2based therapy. We report here two cases of metastatic CXPA that were treated with trastuzumab-based chemotherapy (IRB approved) with rapid and significant responses. Case Report 1: A 66-year-old male was diagnosed as HER2-positive CXPA of the right lacrimal gland with multiple bone and lymph node metastases. Combination chemotherapy with trastuzumab (Tmab) and nanoparticle albumin-bound paclitaxel (nabPTX) was initiated. A rapid response was confirmed, and after seven cycles of treatment, CR(complete response) was achieved. Case Report 2: A 67-year-old female was diagnosed with HER2 positive CXPA of the right submandibular gland. Multiple pulmonary metastatic lesions were detected after surgery, and combination chemotherapy with Tmab and nab-PTX was initiated. A rapid partial response (PR) was confirmed, and she eventually became disease-free. Conclusion: In the absence of definitive clinical trials, which are unlikely to be performed due to the rarity of HER2-positive CXPA, therapeutic information must be obtained from case reports. Some reports, such as this one, have suggested a potential utility of trastuzumab-based chemotherapy.

(C) 2018 The Author(s)

Published by S. Karger AG, Basel

\section{Introduction}

Carcinoma ex pleomorphic adenoma (CXPA) is a rare histologic subtype of submandibular gland and lacrimal gland cancer [1]. This tumor predominantly affects the parotid gland and accounts for $11 \%$ of salivary gland malignancies [2]. On the other hand, only a few primary lacrimal CXPA have been reported [3].

Currently, there is no standard treatment for metastatic CXPA, although some case reports have explored the role of targeted agents in chemotherapy [4, 5]. A histopathologic analysis of 31 cases of CXPA showed that some of these tumors overexpress human epidermal growth factor receptor-2 (HER2) [6]. CXPA in the minimally invasive phase has an excellent prognosis regardless of histological subtype or molecular factor [7]. However, advanced or metastatic CXPA have a poor prognosis, and HER2 overexpression is significantly associated with a worse prognosis in advanced CXPA [6]. These data suggest a potential utility for HER2targeted chemotherapy against this subtype of tumor.

We report here two cases of metastatic CXPA of the lacrimal gland and submandibular gland, respectively, that were treated with trastuzumab (Tmab; an anti HER2 monoclonal antibody) combined with nabPTX chemotherapy (IRB approved) with rapid and significant responses.

\section{Case Report 1}

A 66-year-old male noticed exacerbation of right exophthalmos and diplopia a few months before his initial visit to our institution. Head computed tomography (CT) and magnetic resonance imaging (MRI) revealed a tumor in the right orbit. Tumorectomy of the right lacrimal gland with enucleation was performed. Histologically, the tumor consisted of broad 
Arihara et al.: Trastuzumab-Based Combination Chemotherapy in Patients with Human Epidermal Growth Factor Receptor-2-Positive Metastatic Carcinoma ex Pleomorphic Adenoma

hyalinized stroma with nests of tumor cells, low atypical ductal structure background and a pleomorphic adenoma-component (Fig. 1A, B). The resected tumor showed strong positivity for HER2 immunostaining (score 3+, Fig. 1C). Consequently, the patient was diagnosed with HER2-positive CXPA of the lacrimal grand. After surgery, positron emission tomography (PET)-CT was performed, which revealed multiple bone and lymph node metastatic lesions. Then, combination chemotherapy with Tmab $(8 \mathrm{mg} / \mathrm{kg}$ dose loading, followed by $6 \mathrm{mg} / \mathrm{kg}$ every three weeks) and nab-paclitaxel (nabPTX; $220 \mathrm{mg} / \mathrm{m}^{2}$ every three weeks) was initiated. A rapid partial response was confirmed after only two treatment cycles without any severe adverse effects. After seven cycles, PET-CT revealed no evidence of residual tumor and CR was achieved (Fig. 2). The patient was maintained with trastuzumab monotherapy without disease recurrence. After 6 months of Tmab maintenance therapy, we decided to terminate the Tmab administration. He continues to be disease-free more than two years after the initiation of Tmab and nabPTX combination therapy.

\section{Case Report 2}

A 67-year-old female noticed enlargement of a right submandibular nodule, which had existed for more than 30 years, and visited a local hospital. Head and neck CT and MRI revealed an irregularly shaped, approximately $7 \times 5 \mathrm{~cm}$ tumor in her right submandibular gland. A total right submandibular-glandectomy was performed. The tumor consisted of a salivary duct carcinoma (SDC) component with low atypical ductal structures and hyalinized stroma. On the basis of the above information, she was diagnosed as CXPA with an SDC component of the right submandibular gland. The resected tumor showed strong positivity for HER2 (score $3+$ ). Thereafter, combination chemotherapy with Tmab and nabPTX was initiated. A rapid partial response was confirmed after only one treatment cycle, without any severe toxicity. The best overall response (PR, -76\%) was achieved after eight cycles of treatment (Fig. 3). However, she suffered from CTCAE(Common Terminology Criteria for Adverse Events v4.0) grade 2 peripheral neutropenia, induced by the eight courses of nabPTX treatment. We decided then to exclude nabPTX and continue only with Tmab as a maintenance therapy. We continued the HER2-based chemotherapy for more than a year, and performed a partial lung resection to remove the only remaining lung tumor. The patient has remained disease-free without recurrence for more than two years after initiation of nabPTX and Tmab combination therapy.

\section{Discussion}

Salivary gland cancers and lacrimal gland cancers comprise a heterogenous group of rare tumors. Especially, CXPA is a rare histologic subtype of these head and neck tumors. A certain population of CXPA is known to overexpress HER2 and could potentially benefit from the treatment with HER2-targeted agents [6].

Tmab was one of the first molecular-targeted drugs to be developed and was originally introduced for the treatment of HER2-positive metastatic breast cancer [8]. Based on the findings accumulated from the treatment of breast cancer, Tmab is now considered a key drug for treating HER2-positive malignancies. 
In a phase II trial of Tmab monotherapy in fourteen patients with advanced HER2-positive salivary gland carcinomas, only one partial response (7.1\%) was achieved [9]. However, a handful of case series have demonstrated dramatic responses to Tmab-based combination chemotherapy regimens against HER2-positive SDC, including CXPA, suggesting the potential utility of Tmab combination therapy (Table 1) [4, 5, 10,11].

As the anti-cancer agent to combine with Tmab, we selected nabPTX, an albumin-bound form of paclitaxel, which was solvent-free and designed to improve the therapeutic index of paclitaxel [12]. Taxanes are among the most widely used chemotherapy agents in the treatment of malignant tumors, including breast, lung, and gastric cancer [13-15]. In addition, recent studies have shown clinical efficacy of taxane-trastuzumab combination regimens in HER2-positive cancer [8]. Therefore, we selected nabPTX as the partner in Tmab combination therapy.

In the present study, we have presented two cases of HER2-positive metastatic CXPA derived from the salivary gland and lacrimal gland, respectively. Combination treatment with Tmab and nabPTX demonstrated a promising anti-tumor effect without any severe adverse effects.

In the absence of definitive clinical trials, which are unlikely to be performed due to the rarity of this tumor, we believe that our case report suggests a potential utility of Tmab-based chemotherapy for HER2-positive CXPA.

\section{Acknowledgement}

None.

\section{Statement of Ethics}

This Tmab and nabPTX regimen was administered to HER-2-positive CXPA patients with the approval of the Sapporo Medical University IRB committee and the approval of the treated patients in accordance with the Declaration of Helsinki.

\section{Disclosure Statement}

The authors declare no competing financial interests to disclose.

\section{Author Contributions}

YAr and KM wrote the manuscript. KT supervised the paper preparation and patient care. $\mathrm{NH}$ and SM were physicians in charge of patient care. SS provided pathology reports. MKu and YAk did surgical procedures. KM, MKo and JK performed a critical review. 
Arihara et al.: Trastuzumab-Based Combination Chemotherapy in Patients with Human Epidermal Growth Factor Receptor-2-Positive Metastatic Carcinoma ex Pleomorphic Adenoma

\section{References}

1 Lewis JE, Olsen KD, Sebo TJ. Carcinoma ex pleomorphic adenoma: pathologic analysis of 73 cases. Hum Pathol. 2001 Jun;32(6):596-604.

2 Gnepp DR. Malignant mixed tumors of the salivary glands: a review. Pathol Annu. 1993;28(Pt 1):279-328.

3 Kini YK, Halli R, Mishra S, Kalburge JV. Comprehensive management of a rare carcinoma ex pleomorphic adenoma of the lacrimal gland with a modified lateral orbitotomy access osteotomy. A case report. Oral Maxillofac Surg. 2012 Mar;16(1):123-6.

4 Kadowaki S, Yatabe Y, Hirakawa H, Komori A, Kondoh C, Hasegawa Y, et al. Complete Response to Trastuzumab-Based Chemotherapy in a Patient with Human Epidermal Growth Factor Receptor-2-Positive Metastatic Salivary Duct Carcinoma ex Pleomorphic Adenoma. Case Rep Oncol. 2013 Sep;6(3):450-5.

5 Sharon E, Kelly RJ, Szabo E. Sustained response of carcinoma ex pleomorphic adenoma treated with trastuzumab and capecitabine. Head Neck Oncol. 2010 May;2(1):12.

6 Hashimoto K, Yamamoto H, Shiratsuchi H, Nakashima T, Tamiya S, Nishiyama K, et al. HER-2/neu gene amplification in carcinoma ex pleomorphic adenoma in relation to progression and prognosis: a chromogenic in-situ hybridization study. Histopathology. 2012 May;60 6B:E131-42.

7 Di Palma S, Skálová A, Vanìèek T, Simpson RH, Stárek I, Leivo I. Non-invasive (intracapsular) carcinoma ex pleomorphic adenoma: recognition of focal carcinoma by HER-2/neu and MIB1 immunohistochemistry. Histopathology. 2005 Feb;46(2):144-52.

8 Slamon DJ, Leyland-Jones B, Shak S, Fuchs H, Paton V, Bajamonde A, et al. Use of chemotherapy plus a monoclonal antibody against HER2 for metastatic breast cancer that overexpresses HER2. N Engl J Med. 2001 Mar;344(11):783-92.

9 Haddad R, Colevas AD, Krane JF, Cooper D, Glisson B, Amrein PC, et al. Herceptin in patients with advanced or metastatic salivary gland carcinomas. A phase II study. Oral Oncol. 2003 Oct;39(7):724-7.

10 Hassanieh I, Hilal L, Al Feghali KA, Khalifeh I, Youssef B. Trastuzumab Emtansine for the Treatment of HER-2 Positive Carcinoma Ex-pleomorphic Adenoma Metastatic to the Brain: A Case Report. Front Oncol. 2018 Jul;8:274.

11 Thorpe LM, Schrock AB, Erlich RL, Miller VA, Knost J, Le-Lindqwister N, et al. Significant and durable clinical benefit from trastuzumab in 2 patients with HER2-amplified salivary gland cancer and a review of the literature. Head Neck. 2017 Mar;39(3):E40-4.

12 Desai N, Trieu V, Yao Z, Louie L, Ci S, Yang A, et al. Increased antitumor activity, intratumor paclitaxel concentrations, and endothelial cell transport of cremophor-free, albumin-bound paclitaxel, ABI-007, compared with cremophor-based paclitaxel. Clin Cancer Res. 2006 Feb;12(4):1317-24.

13 Sasaki Y, Nishina T, Yasui H, Goto M, Muro K, Tsuji A, et al. Phase II trial of nanoparticle albumin-bound paclitaxel as second-line chemotherapy for unresectable or recurrent gastric cancer. Cancer Sci. 2014 Jul;105(7):812-7.

14 Sparano JA, Wang M, Martino S, Jones V, Perez EA, Saphner T, et al. Weekly paclitaxel in the adjuvant treatment of breast cancer. N Engl J Med. 2008 Apr;358(16):1663-71.

15 Kelly K, Crowley J, Bunn PA Jr, Presant CA, Grevstad PK, Moinpour CM, et al. Randomized phase III trial of paclitaxel plus carboplatin versus vinorelbine plus cisplatin in the treatment of patients with advanced non—small-cell lung cancer: a Southwest Oncology Group trial. J Clin Oncol. 2001 Jul;19(13):3210-8. 


\section{Case Reports in Oncology}

Case Rep Oncol 2018;11:835-841

DOI: $10.1159 / 000495344$

(c) 2018 The Author(s). Published by S. Karger AG, Basel www.karger.com/cro

Arihara et al. Trastuzumab-Based Combination Chemotherapy in Patients with Human Epidermal Growth Factor Receptor-2-Positive Metastatic Carcinoma ex Pleomorphic Adenoma
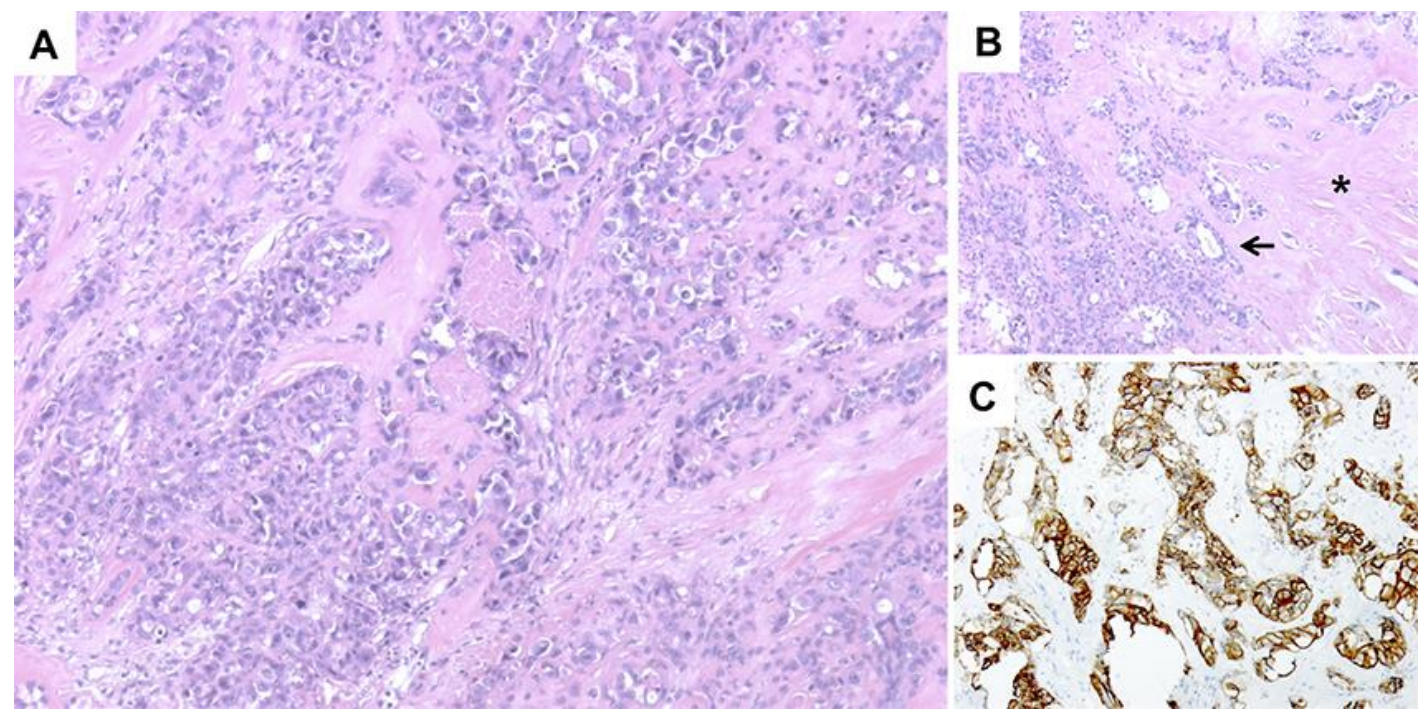

Fig. 1. Histopathological Findings of the Tumor Derived from Case 1. (A) Carcinomatous component $(\times 100$ magnification). (B) Low atypical ductal structure (arrow) and hyalinized stroma (asterisk), hematoxylin and eosin staining ( $\times 200$ magnification). (C) Immunohistochemical analysis of HER2 staining of the tumor ( $\times 200$ magnification). The tumor cells were strongly positive for HER2 (Score $3+$ ).

\section{Baseline}

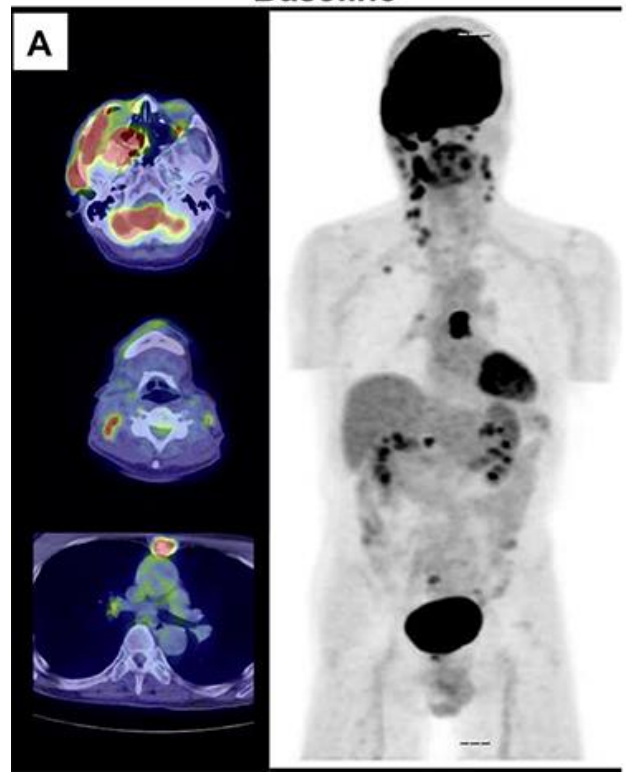

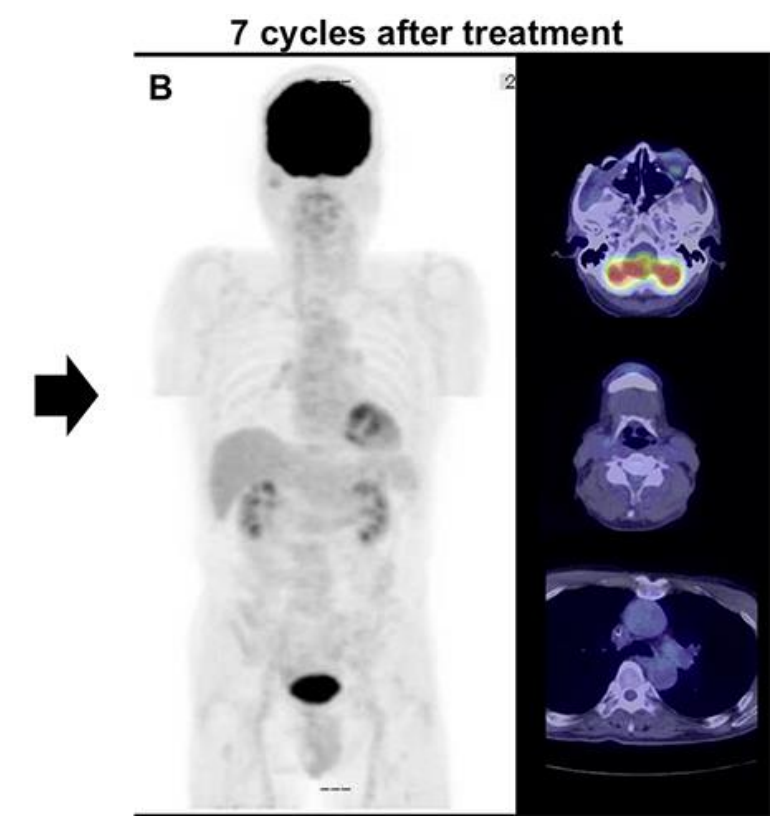

Fig. 2. Clinical Course of Case 1. (A) PET-CT scan revealed local recurrence, multiple bone and lymph node metastases (Baseline). (B) A complete response was achieved after seven cycles of Tmab + nabPTX treatment. 


\section{Case Reports in Oncology}

Case Rep Oncol 2018;11:835-841

DOI: $10.1159 / 000495344$

(C) 2018 The Author(s). Published by S. Karger AG, Basel www.karger.com/cro

Arihara et al.: Trastuzumab-Based Combination Chemotherapy in Patients with Human Epidermal Growth Factor Receptor-2-Positive Metastatic Carcinoma ex Pleomorphic Adenoma
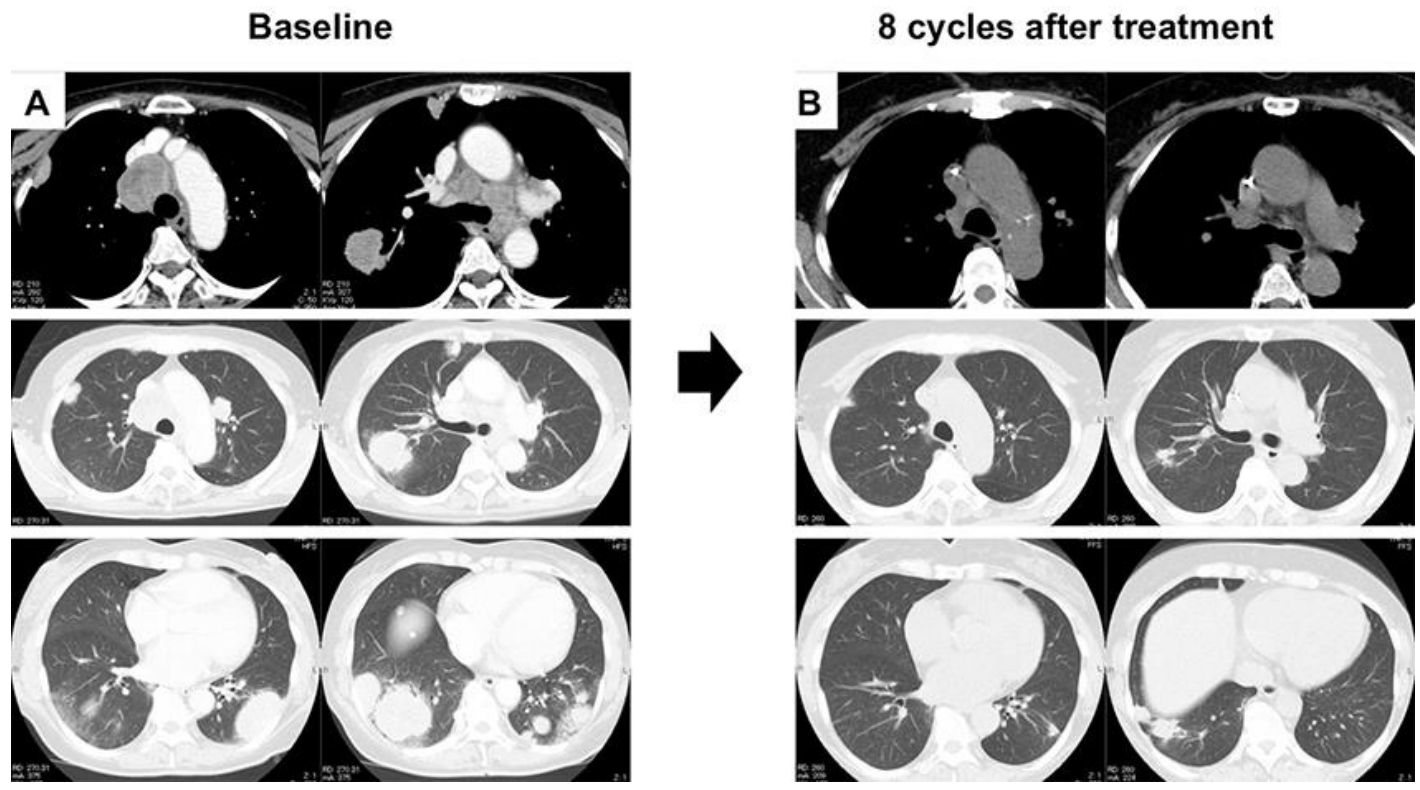

Fig. 3. Clinical Course of Case 2. (A) Thoracic CT scan revealed multiple pulmonary and lymph node metastases (Baseline). (B) A partial response (-76\%) was observed after eight cycles of Tmab + nabPTX treatment.

Table 1. Review of Literature -Tmab Combination Therapy for CXPA or SDC

\begin{tabular}{lrlll}
\hline Author & $\begin{array}{l}\text { Patients, } \\
n\end{array}$ & Pathology & Regimen & Response \\
\hline Hadda et al: Oral Oncol 2003 & 14 & SDC & Tmab monotherapy & CR0, PR1 (7\%) \\
Prat et al: Head Neck 2008 & 1 & SDC & CBDCA/PTX+Tmab & CR \\
Nashed et al: J Laryngol Otol 2009 & 1 & SDC (CXPA) & DTX+Tmab & CR \\
Sharon et al: Head Neck Oncol 2010 & 1 & CXPA & Capecitabine+Tmab & CR \\
Kaidar-Person et al: Med Oncol 2012 & 1 & SDC & CBDCA/PTX+Tmab & CR \\
Kadowaki et al: Case Rep Oncol 2013 & 1 & SDC (CXPA) & PTX+Tmab & CR \\
Thorpe et al: Head Neck 2017 & 2 & CXPA, SDC & PTX+Tmab, chemo+Tmab & CR1, PR1 \\
Hassanieh et al: Front Oncol 2018 & 1 & CXPA & TDM1 & CR1, PR1 \\
\hline Our Cases & 2 & CXPA 2 & nabPTX+Tmab & C
\end{tabular}

CR, complete response; PR, partial response; CBDCA, carboplatin; PTX, paclitaxel; DTX, docetaxel; TDM1, Trastuzumab Emtansine. 